\title{
Trends in the contemporary incidence of colorectal cancer and patient characteristics in the United Kingdom: a population-based cohort study using The Health Improvement Network
}

\author{
Lucía Cea Soriano ${ }^{1,2}$, Montse Soriano-Gabarró ${ }^{3^{*}}$ and Luis A. García Rodríguez ${ }^{1}$
}

\begin{abstract}
Background: Cancer registry data show that survival of colorectal cancer (CRC) in the United Kingdom is poor compared with other European countries and the United States, yet these data sources lack information on patient comorbidities and medication use, which could help explain these differences.

Methods: Among individuals aged 40-89 years in The Health Improvement Network (2000-2014), we identified first ever cases of CRC and calculated incidence rates with 95\% confidence intervals (CIs). For CRC cases and noncases in two separate calendar years (2002 and 2014), we evaluated patient demographics, lifestyle factors, comorbidities and medication use and bowel screening.

Results: The incidence of CRC remained relatively constant across the study period; incidence rates per 10,000 personyears (95\% Cls) were $9.27(8.59-1.01)$ in 2000, 10.65 (10.15-11.18) in 2007 and 8.37 (7.93-8.83) in 2014. Incidence rates per 10,000 person-years were higher in men than women at 11.44 (95\% Cl: 10.35-12.66) vs. 7.40 (95\% Cl: 6.59-8.32) in 2000, and 9.39 (95\% Cl: 8.74-10.10) vs. 7.38 (95\% Cl: 6.81-8.00) in 2014. An increase was seen in the proportion of CRC cases diagnosed at age $<60$ years. In 2002, 3.5\% of CRC cases were diagnosed at age 40-49 compared with 5.1\% in $2014(p=0.064)$. Similarly, in 2002, 12.5\% were diagnosed at age 50-59 years compared with $16.2 \%$ in 2014 ( $p=0.002$ ). Between 2002 and 2014, previous bowel screening increased in both CRC cases (+ 10.6\%) and non-cases $(+9.7 \%)(p<0.001$ for both groups). Greater rises in the following were seen among CRC cases compared with noncases: diabetes (+ 9.3\% vs. $+3.3 \% ; p<0.001$ for both), obesity (+ 14.5\% vs. $+10.1 \% ; p<0.001$ for both), hypertension ( $+8.3 \%$ vs. $+3.6 \% ; p<0.001$ for both), atrial fibrillation ( $+2.6 \%[p<0.01]$ vs. $+0.3 \%[p<0.001])$, and use of proton pump inhibitors (+ 11.5\% vs. $+9.0 \%)$, anti-hypertensives (+ $9.9 \%$ vs. $+1.4 \%)$ and warfarin $(+3.2 \%$ vs. $+0.4 \%) ; p<0.001$ for CRC cases and non-cases with respect to each medication.
\end{abstract}

Conclusions: CRC incidence has remained relatively stable in the UK over the last decade. The increased prevalence of some comorbidities and medications among CRC cases should be considered when evaluating patterns in CRC survival.

Keywords: Colorectal cancer, Incidence, United Kingdom, Comorbidities

* Correspondence: montse.soriano-gabarro@bayer.com

${ }^{3}$ Epidemiology, Bayer AG, 13353 Berlin, Germany

Full list of author information is available at the end of the article 


\section{Background}

In Europe, colorectal cancer (CRC) is the third most commonly diagnosed cancer in males and the second most commonly diagnosed cancer in females [1]. In the United Kingdom (UK), approximately 100 new cases of CRC are diagnosed each day [2]. Cancer registry data show that $\mathrm{CRC}$ incidence rates in the UK have remained relatively stable for over a decade [3] yet improvement in survival has been small [4]. Furthermore, survival rates in the UK have been poor compared with most other countries in Western and Central Europe [5, 6], Scandinavia [5, 6] and the US [7] with a relatively higher number of excess deaths in the first few months following diagnosis $[8,9]$ particularly among older age groups $[9,10]$.

While cancer registries are suitable for determining population-level incidence and survival - including adjustment for age, stage at diagnosis and socio-economic status - data such as the prevalence of comorbidities, medication use before cancer onset and other potential confounders are not systematically recorded. This hinders adjustment for these factors and limits the robustness of comparisons that can be made when making comparisons of CRC survival between populations. In the UK, prospectively collected data on patient comorbidities, prescribed medications and lifestyle factors can be ascertained from primary care databases of electronic medical records (EMRs). Using a validated UK primary care database, we conducted a retrospective cohort study to evaluate trends in the contemporary incidence of CRC in the UK and characteristics preceding CRC diagnosis, including specific comorbidities and medication use. The study protocol was approved by an independent scientific review committee (reference number 14088A1).

\section{Methods}

\section{Data source}

We used data from The Health Improvement Network (THIN), a primary care database of anonymized EMRs in the UK covering approximately $6 \%$ of the UK population [11]. The database is representative of the UK population with regards to age, sex and geographic distribution, and has been validated for use in pharmacoepidemiologic research $[12,13]$. Further details describing THIN can been found in the Additional file 1.

\section{Study population and CRC case identification}

Annually, from 2000 to 2014, we identified all individuals in THIN aged 40-89 years with a registration status of permanent or died. To enter the study, individuals were required to have no previous record of any type of cancer and at least 1 years of enrolment with their primary care practitioner (PCP). All members of the study population were followed-up from the date of entry into the study year (start date) until a first recorded diagnosis for CRC, aged 90 years, death or the end of the calendar year (annually), whichever came first. Individuals with a first recorded diagnosis for CRC during follow-up were deemed to be incident cases of CRC. No additional validation step of CRC cases, such as manual review of patient records or validation with the PCP via questionnaires, was performed because we have previously shown the recording of CRC in THIN to have a high level of validity and completeness - using linkage to hospitalization data, the positive predictive value for CRC in THIN was $97.9 \%(556 / 568)$ and the false negative rate was $6.1 \%(36 / 592)$ [14].

\section{Covariates}

For CRC cases in two separate calendar years approximately a decade apart (2002 [N=931] and 2014 [N=1330]), we obtained data on patient demographics (age, sex, Townsend deprivation score and urban/rural setting), lifestyle factors, healthcare use (number of PCP visits, referrals and hospitalizations), gastrointestinal comorbidities and symptoms, bowel screening procedures (colonoscopy, sigmoidoscopy, barium enema, participation in the National Bowel Screening programme), other comorbidities (with a focus on cardiovascular conditions) and medications. Lifestyle factors and BMI were ascertained any time before the start date, using the most recent value/record. Comorbidities (including gastrointestinal symptoms and bowel screening) were ascertained within the 5 years before the start date. Healthcare use was ascertained in the year before the start date, and medication use was defined as use on the start date or within the previous 30 days. For comparison, we also ascertained these data for all non-CRC cases in $2002(N=1,126,644)$ and $2014(N=1,758,198)$. For each study year this comprised all individuals who did not have a first recorded diagnosis for CRC during follow-up.

\section{Statistical analysis}

We calculated incidence rates of CRC with 95\% confidence intervals (CIs) for each calendar year in the study period using Poisson regression, censoring at the occurrence of another type of cancer. Incidence rates were calculated as the number of first-ever cases of CRC per 10,000 person-years, for the total study population and stratified by sex and 10-year age group. For patient characteristics in 2002 and 2014, data were expressed as frequency counts and percentages and differences compared using Chi2 test for categorical variables, apart from the mean age at the start date, which was presented along with its standard deviation (SD).

\section{Results}

The annual incidence of CRC per 10,000 person-years remained relatively constant across the study period; 
incidence rates increased from 9.27 (95\% CI: 8.59-1.01) in 2000 to 10.65 (95\% CI: 10.15-11.18) in 2007, followed by a decreased trend during the later years in the study period, falling to 8.37 (95\% CI: 7.93-8.83) in 2014 (Fig. 1a). In men, the incidence of CRC per 10,000 person-years was 11.44 (95\% CI: $10.35-12.66$ ) in 2000 and 9.39 (95\% CI: 8.74-10.10) in 2014; corresponding rates for women were 7.40 (95\% CI: 6.59-8.32) and 7.38 (95\% CI: 6.81-8.00). Incidence rates by age at diagnosis across the study period are shown in Fig. $1 \mathrm{~b}$ for men and Fig. 1c for women. The declining incidence of CRC in the later years of the study period was mainly driven by decreasing rates in older men (aged $\geq 60$ years) from 2012, while overall rates in women remained relatively stable during these study years. As shown in Table 1, incidence

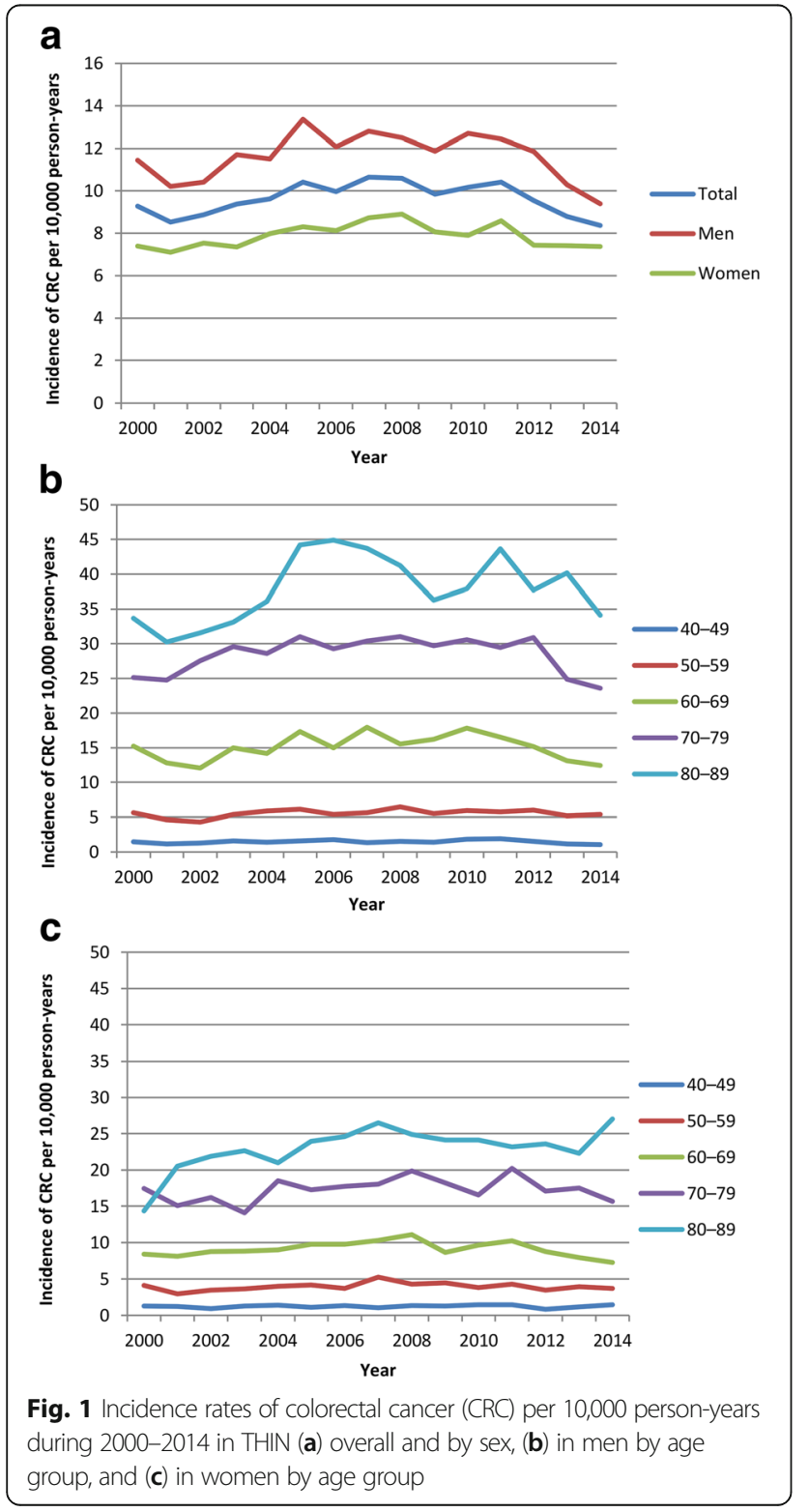

rates of CRC were of similar magnitude and followed a similar trend to those reported by the Office for National Statistics (ONS) for the UK over the same study period. Rates were slightly higher in THIN but this could be owing to the fact that our study population was among adults aged 40-89 years while the ONS rates are among all individuals.

\section{Characteristics of incident CRC cases and non-cases in 2002 and 2014}

Demographics, lifestyle factors and healthcare use among CRC cases and non-cases in 2002 and 2014 are shown in Table 2. No substantial change was seen in the proportion of male and female cases of incident CRC between the two study years $(p=0.711)$. A rise was seen in the proportion of $C R C$ cases diagnosed at ages $<60$ years; in $2002,3.5 \%$ of CRC cases were diagnosed at age $40-49$ compared with $5.1 \%$ in $2014(p=0.064)$. Similarly, in 2002, 12.5\% were diagnosed at age 50-59 years compared with $16.2 \%$ in $2014(p=0.002)$. The mean age at diagnosis was 70.2 years (SD: 10.2) in 2002 and 69.4 years (SD: 11.2) in 2014. Corresponding data (age at start date) for non-cases was 58.6 (SD: 12.8) in 2002 and 57.6 (SD: 12.6) in 2014. Between 2002 and 2014, the proportion of current smokers among CRC cases decreased by $3.1 \%$, with a similar decrease seen among non-cases $(-2.5 \%) \quad(p<0.001$ for both). A decline in nondrinkers was seen in both cases and non-cases $(p<0.001)$. While obesity (BMI $\left.\geq 30 \mathrm{~kg} / \mathrm{m}^{2}\right)$ was recorded more frequently in 2014 than in 2002 in both groups $(p<0.001)$, the increase was greater among CRC cases than for noncases $(+14.5 \%$ vs. $+10.1 \%)$.

Gastrointestinal comorbidities, symptoms and investigative procedures among CRC cases and non-cases in 2002 and 2014 are shown in Table 3. As expected, an increase was seen in the number of individuals with a prior record of participation in the National Bowel Screening programme $(+10.6 \%$ in CRC cases, $p<0.001$ and $+9.7 \%$ in non-cases, $p<0.001)$. The proportion of cases with a record of colonoscopy also increased among CRC cases $(2.8 \%$ to $6.2 \%, p<0.001)$ and in noncases $(1.6 \%$ to $4.2 \%, p<0.001)$. The prevalence of sigmoidoscopy was similar across the study years in cases $(p=0.412)$ but increased among non-cases $(p<0.001)$, while the prevalence of barium enema decreased among both groups ( $p<0.001$ for both).

As shown in Table 4, the prevalence of diabetes, hypertension, atrial fibrillation increased between the two study years in both CRC cases and non-cases, but with a greater increase seen in cases: $+8.8 \%(p<0.001)$ vs. $+6.5 \%(p<0.001)$ for diabetes, $+8.3 \%(p<0.001)$ vs. $+3.6 \%(p<0.001)$ for hypertension, and $+2.6 \%(p<0.01)$ vs. $+0.3 \%(p<0.001)$ for atrial fibrillation. This trend was also seen with use of proton pump inhibitors (PPIs), 
Table 1 Annual incidence rates of CRC per 10,000 person-years in the UK (2000-2014): comparison of data from THIN and ONS

\begin{tabular}{|c|c|c|c|c|c|c|c|c|c|c|c|c|c|c|c|}
\hline & \multicolumn{15}{|c|}{ Incidence rates of CRC per 10,000 person-years } \\
\hline & 2000 & 2001 & 2002 & 2003 & 2004 & 2005 & 2006 & 2007 & 2008 & 2009 & 2010 & 2011 & 2012 & 2013 & 2014 \\
\hline \multicolumn{16}{|l|}{ Total } \\
\hline THIN & 9.27 & 8.54 & 8.87 & 9.38 & 9.63 & 10.41 & 9.98 & 10.65 & 10.59 & 9.86 & 10.17 & 10.42 & 9.53 & 8.79 & 8.37 \\
\hline ONS & 7.26 & 7.04 & 6.94 & 6.99 & 7.15 & 7.17 & 7.26 & 7.36 & 7.48 & 7.55 & 7.53 & 7.60 & 7.54 & 7.21 & 7.00 \\
\hline \multicolumn{16}{|l|}{ Males } \\
\hline THIN & 11.44 & 10.20 & 10.41 & 11.71 & 11.51 & 13.37 & 12.07 & 12.80 & 12.49 & 11.88 & 12.71 & 12.44 & 11.85 & 10.30 & 9.39 \\
\hline ONS & 9.23 & 8.96 & 8.79 & 8.96 & 9.09 & 9.12 & 9.12 & 9.21 & 9.44 & 9.49 & 9.48 & 9.45 & 9.46 & 8.95 & 8.61 \\
\hline \multicolumn{16}{|l|}{ Female } \\
\hline THIN & 7.40 & 7.10 & 7.54 & 7.35 & 7.98 & 8.30 & 8.13 & 8.74 & 8.90 & 8.06 & 7.90 & 8.60 & 7.43 & 7.41 & 7.38 \\
\hline ONS & 5.79 & 5.64 & 5.55 & 5.51 & 5.68 & 5.69 & 5.80 & 5.91 & 5.94 & 6.00 & 5.97 & 6.08 & 6.01 & 5.78 & 5.69 \\
\hline
\end{tabular}

Age ranges were $40-89$ years in THIN; all ages in ONS

CRC colorectal cancer, ONS Office for National Statistics, THIN The Health Improvement Network, UK United Kingdom

anti-hypertensives and warfarin use at the start date $(p<0.001$ for all) (Table 5). Current use of low-dose aspirin increased slightly in CRC cases $(p<0.001)$ and decreased slightly in non-cases $(p<0.001)$, while current use of non-steroidal anti-inflammatory drugs decreased in both CRC cases $(p=0.002)$ and non-cases $(p<0.001)$. Statin prescribing notably increased among cases $(+26.8 \%$, $p<0.001)$ and increased but to a lesser extent among noncases $(+13.0 \%, p<0.001)$.

\section{Discussion}

In this large population-based study set in a representative primary care setting, we have described the contemporary epidemiology of CRC in the UK and characterized CRC patients at the time of diagnosis in two calendar years more than a decade apart. Few studies have described changes in the comorbidity profile of CRC patients over time [15] and we are unaware of any other study to describe a wide range of patient comorbidities and medication use.

While we have shown that the incidence of CRC has remained relatively stable in the UK over the last 15 years, incidence rates appear to have declined in more recent years, particularly in men aged $\geq 60$ years. Our study also found increased uptake of the National Bowel Screening programme over the study period, which could explain the finding of a slightly earlier mean age at diagnosis in 2014 compared with 2002. The national faecal occult blood test screening programme was rolled out in England in June 2006 and in Scotland in June 2007 with the aim of reducing the number of incident cases through detecting pre-cancerous CRC adenomas. Primary care practitioners involved in the programme were informed of participants who failed to complete the programme, and this may have contributed to an increase in the detection or reporting of new cases that may have previously gone unnoticed - this could explain the peak in CRC incidence rates in this current study in 2007. The incidence rates of CRC in this present study are in line, albeit slightly higher, with those from UK Cancer Registry data [3] and similar age- and gender-specific trends were seen over the study period.

Several factors should ideally be taken into account when analyzing trends in survival of patients with $\mathrm{CRC}$ across time and when making comparisons with other geographical populations, including the comorbidity profile and medication use of patients at the time of diagnosis. Several studies have shown that high comorbidity levels are associated with poorer survival [10, 16-22]. In the National Cancer Data Repository, Downing et al. [10] showed that $24.2 \%$ of colon cancer patients who died within the first month following diagnosis had a Charlson comorbidity score of $\geq 3$, compared with $17.6 \%, 14.2 \%$ and $7.2 \%$ with a score of two, one or zero, respectively. In a population-based study in Denmark, Erichsen et al. [23] showed that comorbidities interacted with CRC to increase mortality beyond that explained by CRC and comorbidities acting independently, particularly in the first year after CRC diagnosis. In the Netherlands, overall comorbidity among over 27,000 CRC patients in the Eindhoven cancer registry increased from $47 \%$ to $62 \%$ between 1995 and 2010, with hypertension increasing from $16 \%$ to $29 \%$ and cardiovascular disease increasing from $12 \%$ to $24 \%$ [15]. Diabetes, hypertension and atrial fibrillation and obesity, were the main characteristics observed to be more prevalent among CRC cases diagnosed in 2014 than among those diagnosed in 2002. Although these conditions were also more prevalent among non-cases in these two study years, the level of increase was notably higher among CRC cases, especially for diabetes. It is possible that more frequent PCP visits among patients presenting with CRC symptoms, or a higher level of monitoring among high-risk patients, could have led to better recording of other comorbidities and lifestyle factors. However, a 
Table 2 Demographics, lifestyle factors and healthcare use among CRC cases and non-cases in 2002 and 2014

\begin{tabular}{|c|c|c|c|c|c|c|c|c|}
\hline & \multicolumn{2}{|l|}{ CRC cases } & \multirow{3}{*}{$\%$ change } & \multirow{3}{*}{$p$ value } & \multicolumn{2}{|l|}{ Non-cases } & \multirow{3}{*}{$\%$ change } & \multirow{3}{*}{$p$ value } \\
\hline & $2002(N=921)$ & $2014(N=1330)$ & & & $2002(N=1,125,723)$ & $2014(N=1,756,868)$ & & \\
\hline & $n(\%)$ & $n(\%)$ & & & $n(\%)$ & $n(\%)$ & & \\
\hline Sex & & & & 0.711 & & & & $<0.001$ \\
\hline Male & $501(54.4)$ & $734(55.2)$ & +0.8 & & $523,503(46.5)$ & $866,071(49.3)$ & +2.8 & \\
\hline Female & $420(45.6)$ & $596(44.8)$ & -0.8 & & $602,220(53.5)$ & $890,797(50.7)$ & -2.8 & \\
\hline Age at start date ${ }^{a}$ (years) & & & & 0.001 & & & & $<0.001$ \\
\hline $40-49$ & $32(3.5)$ & $68(5.1)$ & +1.6 & & $330,063(29.3)$ & $577,167(32.9)$ & +3.6 & \\
\hline $50-59$ & $115(12.5)$ & $215(16.2)$ & +3.7 & & $305,523(27.1)$ & $464,427(26.4)$ & -0.7 & \\
\hline $60-69$ & $244(26.5)$ & $334(25.1)$ & -1.4 & & $234,955(20.9)$ & $374,036(21.3)$ & +0.4 & \\
\hline $70-79$ & $355(38.5)$ & $421(31.7)$ & -6.8 & & $174,098(15.5)$ & $229,621(13.1)$ & -2.4 & \\
\hline $80-89$ & $175(19.0)$ & $292(22.0)$ & +3.0 & & $81,084(7.2)$ & $111,617(6.4)$ & -0.8 & \\
\hline Smoking & & & & $<0.001$ & & & & $<0.001$ \\
\hline Non-smoker & $393(42.7)$ & $575(43.2)$ & +0.5 & & $511,656(45.5)$ & $841,369(47.9)$ & +2.4 & \\
\hline Current & $145(15.7)$ & $168(12.6)$ & -3.1 & & $229,660(20.4)$ & $314,311(17.9)$ & -2.5 & \\
\hline Former & $230(25.0)$ & $580(43.6)$ & +18.6 & & $190,297(16.9)$ & $577,415(32.9)$ & +16 & \\
\hline Unknown & 153 (16.6) & $7(0.5)$ & -16.1 & & $194,110(17.2)$ & $23,773(1.4)$ & -15.8 & \\
\hline BMI $\left(\mathrm{kg} / \mathrm{m}^{2}\right)$ & & & & $<0.001$ & & & & $<0.001$ \\
\hline $15-19$ & $23(2.5)$ & $48(3.6)$ & +1.1 & & $39,966(3.6)$ & $65,177(3.7)$ & +0.1 & \\
\hline $20-24$ & $253(27.5)$ & $316(23.8)$ & -3.7 & & $316,584(28.1)$ & $482,255(27.4)$ & -0.7 & \\
\hline $25-29$ & $298(32.4)$ & $517(38.9)$ & +6.5 & & $323,628(28.7)$ & $594,699(33.8)$ & +5.1 & \\
\hline$\geq 30$ & $116(12.6)$ & $360(27.1)$ & +14.5 & & $169,036(15.0)$ & $441,310(25.1)$ & +10.1 & \\
\hline Unknown & $231(25.1)$ & $89(6.7)$ & -18.4 & & $276,509(24.6)$ & $173,427(9.9)$ & -14.7 & \\
\hline Polypharmacy & & & & $<0.001$ & & & & $<0.001$ \\
\hline 0-1 medications & $517(56.1)$ & $612(46.0)$ & -10.1 & & $792,376(70.4)$ & $1,151,402(65.5)$ & -4.9 & \\
\hline 2-4 medications & $273(29.6)$ & $357(26.8)$ & -2.8 & & $231,408(20.6)$ & $345,485(19.7)$ & -0.9 & \\
\hline$\geq 5$ medications & $131(14.2)$ & $361(27.1)$ & +12.9 & & $101,939(9.1)$ & $259,981(14.8)$ & +5.7 & \\
\hline Alcohol (u/w) & & & & $<0.001$ & & & & $<0.001$ \\
\hline None & $113(12.3)$ & $230(17.3)$ & +5.0 & & $150,487(13.4)$ & $268,029(15.3)$ & +1.9 & \\
\hline $1-9$ & $392(42.6)$ & $621(46.7)$ & +4.1 & & $471,097(41.8)$ & $785,666(44.7)$ & +2.9 & \\
\hline $10-20$ & 116 (12.6) & $219(16.5)$ & +3.9 & & $142,656(12.7)$ & $297,466(16.9)$ & +4.2 & \\
\hline $21-41$ & $45(4.9)$ & $72(5.4)$ & +0.5 & & $48,314(4.3)$ & $94,073(5.4)$ & +1.1 & \\
\hline$\geq 42$ & $15(1.6)$ & $31(2.3)$ & +0.7 & & $16,227(1.4)$ & $40,976(2.3)$ & +0.9 & \\
\hline Unknown & $240(26.1)$ & $157(11.8)$ & -14.3 & & $296,942(26.4)$ & $270,658(15.4)$ & -11 & \\
\hline PCP visits ${ }^{b}$ & & & & $<0.001$ & & & & $<0.001$ \\
\hline $0-4$ & $374(40.6)$ & $249(18.7)$ & -21.9 & & $622,029(55.3)$ & $582,007(33.1)$ & -22.2 & \\
\hline $5-9$ & $252(27.4)$ & $319(24.0)$ & -3.4 & & $277,673(24.7)$ & $435,570(24.8)$ & +0.1 & \\
\hline $10-14$ & $135(14.7)$ & $242(18.2)$ & +3.5 & & $121,781(10.8)$ & $291,525(16.6)$ & +5.8 & \\
\hline $15-19$ & $79(8.6)$ & $171(12.9)$ & +4.3 & & $54,652(4.9)$ & $177,759(10.1)$ & +5.2 & \\
\hline$\geq 20$ & $81(8.8)$ & $349(26.2)$ & +17.4 & & $49,588(4.4)$ & 270,007 (15.4) & +11 & \\
\hline Referrals ${ }^{\mathrm{b}}$ & & & & $<0.001$ & & & & $<0.001$ \\
\hline $0-1$ & $636(69.1)$ & $531(39.9)$ & -29.2 & & $920,723(81.8)$ & $929,757(52.9)$ & -28.9 & \\
\hline $2-4$ & $218(23.7)$ & $380(28.6)$ & +4.9 & & 155,781 (13.8) & $464,696(26.5)$ & +12.7 & \\
\hline $5-9$ & $58(6.3)$ & $279(21.0)$ & +14.7 & & $41,329(3.7)$ & $261,011(14.9)$ & +11.2 & \\
\hline
\end{tabular}


Table 2 Demographics, lifestyle factors and healthcare use among CRC cases and non-cases in 2002 and 2014 (Continued)

\begin{tabular}{|c|c|c|c|c|c|c|c|c|}
\hline & \multicolumn{2}{|l|}{ CRC cases } & \multirow{3}{*}{$\%$ change } & \multirow{3}{*}{$p$ value } & \multicolumn{2}{|l|}{ Non-cases } & \multirow{3}{*}{$\%$ change } & \multirow{3}{*}{$p$ value } \\
\hline & $2002(N=921)$ & $2014(N=1330)$ & & & \multirow{2}{*}{$\begin{array}{l}2002(N=1,125,723) \\
n(\%)\end{array}$} & \multirow{2}{*}{$\begin{array}{l}2014(N=1,756,868) \\
n(\%)\end{array}$} & & \\
\hline & $n(\%)$ & $n(\%)$ & & & & & & \\
\hline$\geq 10$ & $9(1.0)$ & $140(10.5)$ & +9.5 & & $7890(0.7)$ & $101,404(5.8)$ & +5.1 & \\
\hline Hospitalizations $^{\mathrm{b}}$ & & & & $<0.001$ & & & & $<0.001$ \\
\hline None & $829(90.0)$ & $1109(83.4)$ & -6.6 & & $1,063,618(94.5)$ & $1,557,111(88.6)$ & -5.9 & \\
\hline 1 & $57(6.2)$ & $133(10.0)$ & +3.8 & & $43,745(3.9)$ & $134,083(7.6)$ & +3.7 & \\
\hline 2 & $23(2.5)$ & $49(3.7)$ & +1.2 & & $11,974(1.1)$ & $40,510(2.3)$ & +1.2 & \\
\hline$\geq 3$ & $12(1.3)$ & $39(2.9)$ & +1.6 & & $6386(0.6)$ & $25,164(1.4)$ & +0.8 & \\
\hline Townsend score & & & & $<0.001$ & & & & $<0.001$ \\
\hline Quintile 1 (least deprived) & $225(24.4)$ & $340(25.6)$ & +1.2 & & $304,177(27.0)$ & $466,028(26.5)$ & -0.5 & \\
\hline Quintile 2 & $183(19.9)$ & $329(24.7)$ & +4.8 & & $253,931(22.6)$ & $394,091(22.4)$ & -0.2 & \\
\hline Quintile 3 & $180(19.5)$ & $297(22.3)$ & +2.8 & & $219,425(19.5)$ & $355,598(20.2)$ & +0.7 & \\
\hline Quintile 4 & $183(19.9)$ & $185(13.9)$ & -6.0 & & $178,640(15.9)$ & $289,718(16.5)$ & +0.6 & \\
\hline Quintile 5 (most deprived) & $98(10.6)$ & $140(10.5)$ & -0.1 & & $117,993(10.5)$ & $191,274(10.9)$ & +0.4 & \\
\hline Unknown & $52(5.6)$ & $39(2.9)$ & -2.7 & 0.001 & $51,557(4.6)$ & $60,159(3.4)$ & -1.2 & $<0.001$ \\
\hline \multicolumn{9}{|l|}{ Setting } \\
\hline Urban & $562(61.0)$ & $748(56.2)$ & -4.8 & & $720,972(64.0)$ & $1,086,412(61.8)$ & -2.2 & \\
\hline Town & $120(13.0)$ & $148(11.1)$ & -1.9 & & $129,017(11.5)$ & $178,340(10.2)$ & -1.3 & \\
\hline Rural & $58(6.3)$ & $73(5.5)$ & -0.8 & & $76,435(6.8)$ & $98,160(5.6)$ & -1.2 & \\
\hline Unknown & $181(19.7)$ & $361(27.1)$ & +7.4 & & $199,299(17.7)$ & $393,956(22.4)$ & +4.7 & \\
\hline
\end{tabular}

${ }^{a}$ Entry into the study year (start date)

${ }^{b}$ In the year before the start date

$B M I$ body mass index, $C R C$ colorectal cancer, $P C P$ primary care practitioner, $u / w$ units per week

Table 3 Gastrointestinal comorbidities, symptoms and investigative procedures among CRC cases and non-cases in 2002 and 2014

\begin{tabular}{|c|c|c|c|c|c|c|c|c|}
\hline & \multicolumn{2}{|l|}{ CRC cases } & \multirow{3}{*}{$\%$ change } & \multirow[t]{3}{*}{$p$-value } & \multicolumn{2}{|l|}{ Non-cases } & \multirow{3}{*}{$\%$ change } & \multirow[t]{3}{*}{$p$-value } \\
\hline & $2002(N=921)$ & $2014(N=1330)$ & & & $2002(N=1,125,723)$ & $2014(N=1,756,868)$ & & \\
\hline & $n(\%)$ & $n(\%)$ & & & $n(\%)$ & $n(\%)$ & & \\
\hline Bleeding per rectum & $79(8.6)$ & $73(5.5)$ & -3.1 & 0.004 & $22,989(2.0)$ & $38,394(2.2)$ & +0.2 & $<0.001$ \\
\hline Change in bowel habits & $26(2.8)$ & $54(4.1)$ & +1.3 & 0.119 & $12,190(1.1)$ & $29,360(1.7)$ & +0.6 & $<0.001$ \\
\hline Abnormal weight loss & $19(2.1)$ & $26(2.0)$ & -0.1 & 0.857 & $7972(0.7)$ & $20,924(1.2)$ & +0.5 & $<0.001$ \\
\hline Gl adenoma & $20(2.2)$ & $41(3.1)$ & +0.9 & 0.191 & $5035(0.4)$ & $16,565(0.9)$ & +0.5 & $<0.001$ \\
\hline GORD & $48(5.2)$ & $88(6.6)$ & +1.4 & 0.169 & $55,237(4.9)$ & $110,752(6.3)$ & +1.4 & $<0.001$ \\
\hline $\begin{array}{l}\text { Complicated/uncomplicated } \\
\text { PU }\end{array}$ & $20(2.2)$ & $28(2.1)$ & -0.1 & 0.915 & $13,891(1.2)$ & $13,544(0.8)$ & -0.4 & $<0.001$ \\
\hline Complicated PU & $9(1.0)$ & $23(1.7)$ & +0.7 & 0.138 & $6994(0.6)$ & $9015(0.5)$ & -0.1 & $<0.001$ \\
\hline Uncomplicated PU & $13(1.4)$ & $8(0.6)$ & -0.8 & 0.049 & $7977(0.7)$ & $5484(0.3)$ & -0.4 & $<0.001$ \\
\hline $\mathrm{IBD}$ & $19(2.1)$ & $25(1.9)$ & -0.2 & 0.700 & $26,919(2.4)$ & $33,242(1.9)$ & -0.5 & 0.032 \\
\hline $\begin{array}{l}\text { National bowel } \\
\text { screening programme }\end{array}$ & $34(3.7)$ & $190(14.3)$ & +10.6 & $<0.001$ & $<0.001$ & $187,548(10.7)$ & +9.7 & $<0.001$ \\
\hline Colonoscopy & $26(2.8)$ & $83(6.2)$ & +3.4 & $<0.001$ & $17,952(1.6)$ & $73,186(4.2)$ & +2.6 & $<0.001$ \\
\hline Sigmoidoscopy & $44(4.8)$ & $54(4.1)$ & -0.7 & 0.412 & $19,980(1.8)$ & $36,673(2.1)$ & +0.3 & $<0.001$ \\
\hline Barium enema & $95(10.3)$ & $14(1.1)$ & -9.2 & $<0.001$ & $45,149(4.0)$ & $9619(0.5)$ & -3.5 & $<0.001$ \\
\hline
\end{tabular}


Table 4 Distribution of cardiovascular and other comorbidities among CRC cases and non-cases in 2002 and 2014

\begin{tabular}{|c|c|c|c|c|c|c|c|c|}
\hline & \multicolumn{2}{|l|}{ CRC cases } & \multirow{3}{*}{$\%$ change } & \multirow[t]{3}{*}{$p$-value } & \multicolumn{2}{|l|}{ Non-cases } & \multirow{3}{*}{$\%$ change } & \multirow[t]{3}{*}{$p$-value } \\
\hline & $2002(N=921)$ & $2014(N=1330)$ & & & \multirow{2}{*}{$\begin{array}{l}2002(N=1,125,723) \\
n(\%)\end{array}$} & \multirow{2}{*}{$\begin{array}{l}2014(N=1,756,868) \\
n(\%)\end{array}$} & & \\
\hline & $n(\%)$ & $n(\%)$ & & & & & & \\
\hline $\mathrm{IHD}$ & $92(10.0)$ & $74(5.6)$ & -4.4 & $<0.001$ & $73,352(6.5)$ & $48,806(2.8)$ & -3.7 & $<0.001$ \\
\hline Hypertension & $225(24.4)$ & $435(32.7)$ & +8.3 & $<0.001$ & $176,647(15.7)$ & $339,158(19.3)$ & +3.6 & $<0.001$ \\
\hline Hypercholesterolaemia & $35(3.8)$ & $70(5.3)$ & +1.5 & 0.106 & $37,220(3.3)$ & $82,270(4.7)$ & +1.4 & $<0.001$ \\
\hline DVT/PE & $33(3.6)$ & $37(2.8)$ & -0.8 & 0.282 & $20,408(1.8)$ & $27,139(1.5)$ & -0.3 & $<0.001$ \\
\hline Heart failure & $30(3.3)$ & $30(2.3)$ & -1.0 & 0.147 & $17,086(1.5)$ & $16,112(0.9)$ & -0.6 & $<0.001$ \\
\hline Atrial fibrillation & $33(3.6)$ & $82(6.2)$ & +2.6 & 0.006 & $20,783(1.8)$ & $36,865(2.1)$ & +0.3 & $<0.001$ \\
\hline $\mathrm{Ml}$ & $16(1.7)$ & $25(1.9)$ & +0.2 & 0.804 & $15,651(1.4)$ & $17,206(1.0)$ & -0.4 & $<0.001$ \\
\hline Ischaemic stroke & $12(1.3)$ & $20(1.5)$ & +0.2 & 0.692 & $12,953(1.2)$ & $17,983(1.0)$ & -0.2 & $<0.001$ \\
\hline TIA & $15(1.6)$ & $23(1.7)$ & +0.1 & 0.855 & $12,642(1.1)$ & $15,200(0.9)$ & -0.2 & $<0.001$ \\
\hline Haemorrhagic stroke & $3(0.3)$ & $5(0.4)$ & +0.1 & 0.844 & $1418(0.1)$ & $2370(0.1)$ & 0.0 & 0.041 \\
\hline Anaemia & $53(5.8)$ & $77(5.8)$ & 0.0 & 0.972 & $24,250(2.2)$ & $47,283(2.7)$ & +0.5 & $<0.001$ \\
\hline Diabetes & $79(8.6)$ & $231(17.4)$ & +8.8 & $<0.001$ & $60,930(5.4)$ & $155,718(8.9)$ & +6.5 & $<0.001$ \\
\hline Depression & $66(7.2)$ & $86(6.5)$ & -0.7 & 0.515 & $106,405(9.5)$ & $171,204(9.7)$ & +0.2 & $<0.001$ \\
\hline COPD & $44(4.8)$ & $93(7.0)$ & +2.2 & 0.031 & $24,696(2.2)$ & $64,071(3.6)$ & +1.4 & $<0.001$ \\
\hline Asthma & $92(10.0)$ & $157(11.8)$ & +1.8 & 0.177 & $78,615(7.0)$ & 199,049 (11.3) & +4.3 & $<0.001$ \\
\hline Osteoarthritis & $146(15.9)$ & $196(14.7)$ & -1.2 & 0.468 & $123,356(11.0)$ & $157,758(9.0)$ & -2.0 & $<0.001$ \\
\hline Rheumatoid arthritis & $13(1.4)$ & $14(1.1)$ & -0.3 & 0.442 & $10,383(0.9)$ & $18,234(1.0)$ & +0.1 & $<0.001$ \\
\hline
\end{tabular}

$C R C$ colorectal cancer, COPD chronic obstructive pulmonary disease, DVT deep vein thrombosis, IHD ischaemic heart disease, $M I$ myocardial infarction, $P E$ pulmonary embolism, TIA transient ischaemic attack

Table 5 Principal drug therapies prescribed among CRC cases and non-cases in 2002 and 2014

\begin{tabular}{|c|c|c|c|c|c|c|c|c|}
\hline & \multicolumn{2}{|l|}{ CRC cases } & \multirow[b]{2}{*}{$\%$ change } & \multirow[t]{2}{*}{$p$-value } & \multicolumn{2}{|l|}{ Non-cases } & \multirow[b]{2}{*}{$\%$ change } & \multirow[t]{2}{*}{$p$-value } \\
\hline & $2002(N=921)$ & $2014(N=1330)$ & & & $2002(N=1,125,723)$ & $2014(N=921)$ & & \\
\hline Low-dose aspirin & $150(16.3)$ & $220(16.5)$ & +0.2 & $<0.001$ & $113,852(10.1)$ & $156,206(8.9)$ & -1.2 & $<0.001$ \\
\hline Clopidogrel & $7(0.8)$ & $40(3.0)$ & +2.2 & $<0.001$ & $4441(0.4)$ & $30,405(1.7)$ & +1.3 & $<0.001$ \\
\hline Dipyridamole & $8(0.9)$ & $7(0.5)$ & -0.4 & 0.326 & $3787(0.3)$ & $4040(0.2)$ & -0.1 & $<0.001$ \\
\hline Warfarin & $29(3.1)$ & $84(6.3)$ & +3.2 & 0.001 & $19,844(1.8)$ & $37,797(2.2)$ & +0.4 & $<0.001$ \\
\hline All NSAIDs & $68(7.4)$ & $57(4.3)$ & -3.1 & 0.002 & $92,727(8.2)$ & $97,302(5.5)$ & -2.7 & $<0.001$ \\
\hline tNSAIDs & $60(6.5)$ & $52(3.9)$ & -2.6 & 0.005 & $79,946(7.1)$ & $91,889(5.2)$ & -1.9 & $<0.001$ \\
\hline Coxibs & $8(0.9)$ & $6(0.5)$ & -0.4 & 0.215 & $13,827(1.2)$ & $5655(0.3)$ & -0.9 & $<0.001$ \\
\hline Insulin & $15(1.6)$ & $40(3.0)$ & +1.4 & 0.037 & $12,532(1.1)$ & $27,067(1.5)$ & +0.4 & $<0.001$ \\
\hline Oral antidiabetics & $36(3.9)$ & $150(11.3)$ & +7.4 & $<0.001$ & $35,496(3.2)$ & $101,716(5.8)$ & +2.6 & $<0.001$ \\
\hline Oral corticosteroids & $23(2.5)$ & $35(2.6)$ & +0.1 & 0.843 & $20,602(1.8)$ & $35,869(2.0)$ & +0.2 & $<0.001$ \\
\hline Inhaled steroids & $66(7.2)$ & $74(5.6)$ & -1.6 & 0.0122 & $54,881(4.9)$ & $78,009(4.4)$ & -0.5 & $<0.001$ \\
\hline Statins & $95(10.3)$ & $493(37.1)$ & +26.8 & $<0.001$ & $82,486(7.3)$ & $355,803(20.3)$ & +13.0 & $<0.001$ \\
\hline Non-statins & $9(1.0)$ & $7(0.5)$ & -0.5 & 0.211 & $5192(0.5)$ & $7766(0.4)$ & -0.1 & 0.018 \\
\hline Antidiarrhoeal medications & $29(3.1)$ & $42(3.2)$ & +0.1 & 0.990 & $13,101(1.2)$ & $32,378(1.8)$ & +0.6 & $<0.001$ \\
\hline Antidepressants & $69(7.5)$ & $149(11.2)$ & +3.7 & 0.003 & $92,058(8.2)$ & $217,632(12.4)$ & +4.2 & $<0.001$ \\
\hline Acetaminophen & $150(16.3)$ & $249(18.7)$ & +2.4 & 0.137 & $123,387(11.0)$ & $211,478(12.0)$ & +1.0 & $<0.001$ \\
\hline PPIs & $97(10.5)$ & $293(22.0)$ & +11.5 & $<0.001$ & $69,225(6.1)$ & $266,005(15.1)$ & +9.0 & $<0.001$ \\
\hline Antihypertensive medications & 409 (44.4) & $722(54.3)$ & +9.9 & $<0.001$ & $316,728(28.1)$ & $519,072(29.5)$ & +1.4 & $<0.001$ \\
\hline
\end{tabular}


genuine greater increase in these conditions among CRC patients could translate into more CRC patients having fewer treatment options and ultimately worse survival. In a large study of older age CRC patients investigating population-attributable risks, a substantial proportion of deaths were attributable to congestive heart failure, diabetes and chronic obstructive pulmonary disease [22]. In our study, a $8.8 \%$ increase in the prevalence of diabetes at the time of CRC diagnosis was observed between study years, although only minor changes in the prevalence of heart failure and chronic obstructive pulmonary disease were seen. In relation to medication use at the time of diagnosis, higher increases in the use of PPIs, anti-hypertensives and warfarin among CRC cases compared with noncases between 2002 and 2014 suggests the growing pill burden among CRC patients, a factor that may also influence treatment options in addition to adherence and outcomes. With an aging general population, noncancer related health status could play an increasingly important role in the survival of cancer patients, highlighting the importance of data such as those obtained in this study.

Strengths of our study include the large sample size representative of the UK population as a whole; Blak et al. [13] have shown both the demographic of individuals and the prevalence of major medical conditions in THIN to be generalisable to the UK. In addition, previous validation work has shown a high level of completeness and validity of the recorded CRC diagnoses in THIN [14]. Although we did not link to cancer registry data in our prior validation study [14] another study comparing incidence rates in THIN with those in a UK national cancer registry found the age- and sex-standardized incidence rates of CRC to be very similar during the latter part of their study period (2005-2007) [24]. In addition, a study using data from the UK Clinical Practice Research Datalink, which contains very similar primary care data to THIN, reported a $98 \%$ PPV for the CRC diagnosis in the primary care data when linked to cancer registrations [25]. A limitation of our study is the possible improvement in recording in THIN over time, resulting in an under-estimation of CRC incidence rates and patient characteristics in the early years of the study period. Indeed, there was a notable improvement in the proportion of both CRC cases and non-cases with a recorded BMI, smoking status or alcohol intake between 2002 and 2014. Prior to 2003, PCPs contributing data to THIN did not have specific recording instructions, although any improvements in recording will likely have affected CRC cases and non-cases equally. The year 2003 also saw the introduction of a cancer quality improvement measures in the UK by the NHS, which may not only have improved recording of CRC diagnoses but also of other morbidities and patient characteristics. This would have led to a more complete profile in the patient's records.

Advanced stage at CRC diagnosis is clearly associated with worse survival $[26,27]$, and accounts for the highest proportion of early colon cancer deaths; Downing et al. [10] reported $15.4 \%$ of colon cancer deaths in the month following diagnosis to be Duke's Stage D, compared with approximately $5 \%$ or less for less advanced stages of the disease. There was insufficient information in our dataset to enable us to ascertain Duke's stage of CRC cases at diagnosis and thereby describe any potential changes over time in the proportion of CRC cases diagnosed at advanced stage. Failing to reduce the proportion of CRC cases diagnosed at advanced stage compared with other countries could be a possible explanation for the lower relative survival rates and small improvements in survival rates over the last decade. Diagnostic codes in THIN are not specific for Duke's stage CRC; rather information on CRC histology and stage is entered into patients' medical notes as free-text comments, which were not obtained in this study. We have, however, previously evaluated CRC stage at diagnosis in another study in THIN [28], which included 3033 incident cases of CRC diagnosed between 2000 and 2011 with Duke's stage ascertained through manual review of patients EMRs including the free-text comments. We found that Duke's stage D accounted for $12.7 \%$ of CRC cases diagnosed in 2002 and $16.3 \%$ of CRC cases in 2011, whereas reductions were seen in the proportion of CRC cases diagnosed at Duke's stages A to $C$ between these two calendar years $(6.7 \%$ to $4.1 \%$ for Duke's stage A; $14.4 \%$ to $9.2 \%$ for Duke's stage B; $11.0 \%$ to $8.9 \%$ for Duke's stage C; unpublished data). It should be noted that $55.1 \%$ of the CRC cases in 2002 and $61.4 \%$ of CRC cases in 2011 did not have details on stage recorded in the free text.

\section{Conclusion}

In conclusion, our findings suggest that the increased prevalence of obesity, hypertension, atrial fibrillation and the pill burden among CRC cases should be considered when evaluating and making comparisons in patterns of CRC survival.

\section{Additional file}

\section{Additional file 1: Supplementary Methods. (DOCX $13 \mathrm{~kb}$ )}

\begin{abstract}
Abbreviations
Cl: Confidence interval; EMR: Electronic medical records; PCP: Primary care practitioner; THIN: The Health Improvement Network; UK: United Kingdom
\end{abstract}

\section{Acknowledgements}

We thank EpiMed Communications Ltd. for medical writing assistance funded by Bayer AG. 


\section{Funding}

This study was funded by Bayer AG. The funder had no role in the design of the study, nor the collection, analysis and interpretation of the data nor the interpretation of data and manuscript writing, except for in the form of salary paid to MS-G.

\section{Availability of data and materials}

The datasets generated during and/or analyzed during the current study are available from Dr. Luis A. García Rodríguez upon reasonable request.

\section{Authors' contributions}

LAGR obtained funding, contributed to the study concept and design, acquisition of data, statistical analysis, analysis and interpretation of data, critical revision of the manuscript for important intellectual content, and the decision to submit the article for publication. LCS contributed to study design, acquisition of data, statistical analysis, analysis and interpretation of data, critical revision of the manuscript for important intellectual content, and the decision to submit the article for publication. MSG contributed to the study concept and design, interpretation of data, critical revision of the manuscript for important intellectual content, and the decision to submit the article for publication. All authors read and approved the final manuscript.

\section{Ethics approval and consent to participate}

The study protocol was reviewed and approved by an independent scientific review committee for THIN (reference number), which ensured that data were to be analysed and interpreted appropriately. Data collection for THIN was approved by the South East Multicentre Research Ethics Committee in 2003 and individual studies using THIN data do not require separate ethical approval if only anonymized THIN data is used.

\section{Consent for publication}

Not applicable.

\section{Competing interests}

LCS and LAGR work for CEIFE, which has received a research grant from Bayer AG. LAGR has also served as an advisory board member for Bayer AG. MS-G is a salaried, full-time employee of Bayer AG.

\section{Publisher's Note}

Springer Nature remains neutral with regard to jurisdictional claims in published maps and institutional affiliations.

\section{Author details}

${ }^{1}$ Spanish Centre for Pharmacoepidemiologic Research (CEIFE), Almirante 28, 28004 Madrid, Spain. ${ }^{2}$ Department of Public Health and Maternal and Child Health, Faculty of Medicine, Complutense University of Madrid, Madrid, Spain. ${ }^{3}$ Epidemiology, Bayer AG, 13353 Berlin, Germany.

Received: 25 September 2017 Accepted: 20 March 2018 Published online: 10 April 2018

\section{References}

1. Ferlay J, Steliarova-Foucher E, Lortet-Tieulent J, Rosso S, Coebergh JW, Comber $H$, Forman D, Bray F. Cancer incidence and mortality patterns in Europe: estimates for 40 countries in 2012. Eur J Cancer. 2013:49(6):1374-403.

2. National Institute for Health and Care Excellence. Colorectal cancer: diagnosis and management. NICE guidelines [CG131], November 2011 Updated December 2014 https://www.nice.org.uk/guidance/cg131

3. Cancer Research UK. Bowel cancer incidence trends over time. http://www. cancerresearchuk.org/health-professional/cancer-statistics/statistics-bycancer-type/bowel-cancer/incidence\#heading-Two. Accessed 20 Sep 2017.

4. Cancer Research UK. Bowel cancer survival trends over time. http://www. cancerresearchuk.org/health-professional/cancer-statistics/statistics-bycancer-type/bowel-cancer/survival\#heading-Two. Accessed 20 Sep 2017.

5. Holleczek B, Rossi S, Domenic A, Innos K, Minicozzi P, Francisci S, Hackl M, Eisemann N, Brenner H. On-going improvement and persistent differences in the survival for patients with colon and rectum cancer across Europe 19992007 - results from the EUROCARE-5 study. Eur J Cancer. 2015;51(15):2158-68.

6. Verdecchia A, Francisci S, Brenner H, Gatta G, Micheli A, Mangone L, Kunkler I. Recent cancer survival in Europe: a 2000-02 period analysis of EUROCARE4 data. Lancet Oncol. 2007:8(9):784-96.
7. Ciccolallo L, Capocaccia R, Coleman MP, Berrino F, Coebergh JW, Damhuis RA, Faivre J, Martinez-Garcia C, Moller H, Ponz de Leon M, et al. Survival differences between European and US patients with colorectal cancer: role of stage at diagnosis and surgery. Gut. 2005;54(2):268-73.

8. Engholm G, Kejs AM, Brewster DH, Gaard M, Holmberg L, Hartley R, Iddenden R, Moller H, Sankila R, Thomson CS, et al. Colorectal cancer survival in the Nordic countries and the United Kingdom: excess mortality risk analysis of 5 year relative period survival in the period 1999 to 2000. Int J Cancer. 2007;121(5):1115-22.

9. Morris EJ, Sandin F, Lambert PC, Bray F, Klint A, Linklater K, Robinson D, Pahlman L, Holmberg L, Moller H. A population-based comparison of the survival of patients with colorectal cancer in England, Norway and Sweden between 1996 and 2004. Gut. 2011;60(8):1087-93.

10. Downing A, Aravani A, Macleod U, Oliver S, Finan PJ, Thomas JD, Quirke P, Wilkinson JR, Morris EJ. Early mortality from colorectal cancer in England: a retrospective observational study of the factors associated with death in the first year after diagnosis. Br J Cancer. 2013;108(3):681-5.

11. QuintilesIMS. Medical Research. https://www.sd.imshealth.com/Corporate/ OurSolutions/Medical/Pages/default.aspx.

12. Lewis JD, Schinnar R, Bilker WB, Wang X, Strom BL. Validation studies of the health improvement network (THIN) database for pharmacoepidemiology research. Pharmacoepidemiol Drug Saf. 2007;16(4):393-401.

13. Blak BT, Thompson M, Dattani H, Bourke A. Generalisability of the health improvement network (THIN) database: demographics, chronic disease prevalence and mortality rates. Inform Prim Care. 2011;19(4):251-5.

14. Cea Soriano L, Soriano-Gabarro M, Garcia Rodriguez LA. Validity and completeness of colorectal cancer diagnoses in a primary care database in the United Kingdom. Pharmacoepidemiol Drug Saf. 2016;25(4):385-91.

15. van Leersum NJ, Janssen-Heijnen ML, Wouters MW, Rutten HJ, Coebergh JW, Tollenaar RA, Lemmens VE. Increasing prevalence of comorbidity in patients with colorectal cancer in the south of the Netherlands 1995-2010. Int J Cancer. 2013;132(9):2157-63.

16. van Eeghen EE, Bakker SD, van Bochove A, Loffeld RJ. Impact of age and comorbidity on survival in colorectal cancer. J Gastrointest Oncol. 2015;6(6):605-12.

17. Munro AJ, Bentley AH. Deprivation, comorbidity and survival in a cohort of patients with colorectal cancer. Eur J Cancer Care (Engl). 2004;13(3):254-62.

18. Lemmens VE, Janssen-Heijnen ML, Verheij CD, Houterman S, Repelaer van Driel OJ, Coebergh JW. Co-morbidity leads to altered treatment and worse survival of elderly patients with colorectal cancer. Br J Surg. 2005;92(5):615-23.

19. Gross CP, McAvay GJ, Krumholz HM, Paltiel AD, Bhasin D, Tinetti ME. The effect of age and chronic illness on life expectancy after a diagnosis of colorectal cancer: implications for screening. Ann Intern Med. 2006;145(9):646-53.

20. Rieker RJ, Hammer E, Eisele R, Schmid E, Hogel J. The impact of comorbidity on the overall survival and the cause of death in patients after colorectal cancer resection. Langenbeck's Arch Surg. 2002;387(2):72-6.

21. Ouellette JR, Small DG, Termuhlen PM. Evaluation of Charlson-age comorbidity index as predictor of morbidity and mortality in patients with colorectal carcinoma. J Gastrointest Surg. 2004;8(8):1061-7.

22. Gross CP, Guo Z, McAvay GJ, Allore HG, Young M, Tinetti ME. Multimorbidity and survival in older persons with colorectal cancer. J Am Geriatr Soc. 2006; 54(12):1898-904.

23. Erichsen $R$, Horvath-Puho E, Iversen LH, Lash TL, Sorensen HT. Does comorbidity interact with colorectal cancer to increase mortality? A nationwide population-based cohort study. Br J Cancer. 2013;109(7):2005-13.

24. Haynes K, Forde KA, Schinnar R, Wong P, Strom BL, Lewis JD. Cancer incidence in the health improvement network. Pharmacoepidemiol Drug Saf. 2009;18(8):730-6.

25. Dregan A, Moller H, Murray-Thomas T, Gulliford MC. Validity of cancer diagnosis in a primary care database compared with linked cancer registrations in England. Population-based cohort study. Cancer Epidemiol. 2012;36(5):425-9.

26. Public Health England. National Cancer Intelligence Network. Colorectal Cancer Survival by Stage - NCIN Data Briefing. http://www.ncin.org.uk/ cancer_type_and_topic_specific_work/cancer_type_specific_work/ colorectal_cancer/. Accessed 20 Sep 2017.

27. Maringe C, Walters S, Rachet B, Butler J, Fields T, Finan P, Maxwell R, Nedrebo B, Pahlman L, Sjovall A, et al. Stage at diagnosis and colorectal cancer survival in six high-income countries: a population-based study of patients diagnosed during 2000-2007. Acta Oncol. 2013;52(5):919-32.

28. Garcia Rodriguez LA, Soriano-Gabarro M, Bromley S, Lanas A, Cea Soriano L. New use of low-dose aspirin and risk of colorectal cancer by stage at diagnosis: a nested case-control study in UK general practice. BMC Cancer. 2017;17(1):637. 